\title{
Family Climate and Job Performance: The Role of Family-to-Work Enrichment
}

\author{
Chang Su ${ }^{1, a}$ and Mingjian Zhou ${ }^{2, b}$ \\ ${ }^{1}$ Harbin Institute of Technology, Shenzhen, China \\ ${ }^{2}$ Harbin Institute of Technology, Shenzhen, China \\ a625884582@qq.com, bmngzmj@163.com
}

Keywords: Family climate; Job performance; Family-to-Work Enrichment

\begin{abstract}
This study reviews empirical studies on family-to-work enrichment since the year of 2006, when the theory of work-family enrichment was first proposed. In this article we propose a model and offer a series of research propositions that identify the relationships among family climate, family-to-work enrichment and two dimensions of job performance: task performance and organizational citizenship behavior.
\end{abstract}

\section{Introduction}

Work and family are two important central domains for most employed adults in contemporary society. Many studies investigated how they affect each other negatively. However, work and family can also be reciprocal. For example, a good job provides financial foundation to a satisfied family. On the other hand, a happy family or a good marriage facilities an individual to work hard. Gradually, more and more scholars are transferring research perspective from negative relationship to positive relationship. Nowadays, positive energy is one of themes of modern society, especially in China. Measures of success consist of not only money and power, but also the ability to balance work and family. For individuals to better fulfill personal lives as well as contribute to organizations more effectively, it is necessary to understand how to make work and family benefit each other.

\section{Literature Review of Family-to-Work Enrichment}

Work-family enrichment is defined as the extent to which experiences in one role improve the quality of life in the other role[1]. Enrichment occurs bidirectional: work-to-family enrichment (WFE) and family-to-work enrichment (FWE). FWE is the process through which the positive mood, behaviors, sense of accomplishment and support or resource gains in family domain enhance the quality of work domain. Carlson, et al. [2] developed and validated a 9-item scale to measure FWE. The scale consists of three dimensions: development, affect, and efficiency.

\subsection{Antecedents}

Researchers have examined that enrichment arises from several sources (Table 1).

Table 1. Antecedents of Family-to-Work Enrichment

\begin{tabular}{cll}
\hline \multicolumn{1}{c}{ Level } & \multicolumn{1}{c}{ Antecedents } & \multicolumn{1}{c}{ Support for relationships } \\
\hline \multirow{3}{*}{ Individual } & Family identity & Wayne, Randel et al. (2006) \\
& Core self-evaluations & Bhargava and Baral (2009) \\
& Work engagement & Siu, Lu et al. (2010) \\
\hline \multirow{2}{*}{ Organization } & Supervisor support & Bhargava and Baral (2009);Tang, et al. \\
& Team resources & (2014) \\
& & Hunter, Perry et al. (2010) \\
\hline \multirow{2}{*}{ Family } & Family emotional support & Wayne, Randel et al. (2006) \\
& Family support & Bhargava and Baral (2009) \\
\hline
\end{tabular}

At individual level, FWE is influenced by individuals' family identity [3], core self-evaluations [4] and work engagement [5]. At Family level, FWE arises from the support received in family 
domain [3, 4]. At organization level, FWE can occur when employees experience supervisor support [4] and team resources[6]. The review about antecedents of FWE shows that predictors of FWE tend to come from family domain.

\subsection{Consequences}

FWE is related to work-related, family-related and health-related outcomes [7] (Table 2).

Table 2. Consequences of Family-to-Work Enrichment

\begin{tabular}{cll}
\hline \multicolumn{1}{c}{ Aspects } & \multicolumn{1}{c}{ Consequences } & \multicolumn{1}{c}{ Support for relationships } \\
\hline & $\begin{array}{l}\text { Turnover intentions (-) } \\
\text { Job satisfaction }\end{array}$ & $\begin{array}{l}\text { Wayne, Randel et al. (2006) } \\
\text { Bhargava and Baral (2009); McNall, Nicklin et al. } \\
\text { (2010) } \\
\text { Bhargava and Baral (2009) } \\
\text { Bhargava and Baral (2009); McNall, Nicklin et al. } \\
\text { (2010); Wayne, Casper et al. (2013) } \\
\text { Bhargava and Baral (2009); Hunter, Perry et al. } \\
\text { Affective commitment } \\
\text { Family-related }\end{array}$ \\
$\begin{array}{c}\text { Family satisfaction } \\
\text { Health-related }\end{array}$ & $\begin{array}{l}\text { Physical and mental } \\
\text { health }\end{array}$ & \begin{tabular}{l} 
McNall, Nicklin et al. (2010) \\
\hline
\end{tabular}
\end{tabular}

As to family-related outcomes, family satisfaction is regarded as one outcome of FWE [4, 6]. As to work-related outcomes, some examined that greater FWE [3] related to fewer turnover intentions, but some didn't find the relationship [7]. The explanation is that FWE might not necessarily facilitate behaviors. Another work-related outcome is job satisfaction, which also has mixed findings. Some found a positive relationship between FWE and job satisfaction[4, 7]. However, others didn't find the relationship [6]. These findings are consistent with the study suggesting that individuals prefer to attribute benefits of enrichment to the originating, rather than the receiving domain [8]. The explanation is that employees experiencing spillover of resources from one role into another role tend to increase positive emotion and behavioral investment toward the role providing benefits [8]. Besides outcomes listed above, FWE also leads to affective commitment [4, 7, 9]and OCB [4].

Although studies listed above have achieved success in exploring antecedents and outcomes of FWE, more research is still needed to further understand how and why enrichment happens and what will enrichment brings to individuals' work and family. On one hand, previous studies paid relatively few attention to family's enrichment to work. Future research should explore more on the enrichment effect of family to work. On the other hand, a number of studies were conducted primarily in Western context. But the contexts and culture characteristics of many Asian countries such as China are distinct from those of the West. Therefore, it is worthwhile to explore relationships of work and family in different social, economic and political contexts.

\section{Theoretical Foundation and Conceptual framework}

\subsection{Work-Family Enrichment Model}

The model of work-family enrichment was founded on the following theories. Social exchange theory [10] suggested that when employees perceive help and support by work and family members, they will consequently feel obligated to reward with positive attitudes and behaviors. Conservations of resources (COR) theory [11] suggested that resources in one role can foster gains that facilitate satisfaction or performance in another role. Both theories suggest that individuals' resources are not fixed but actually can be expanded. Investing resources in one domain will result in an increase of resources in the other domain [12]. Role accumulation theory [13] proposed that people choose to participate in multiple roles for various rewards. Benefits accumulated in role performance may cover losses in role involvement. Coincidentally, role expansion theory [14] suggested that role fulfillment can create energy to enhance the fulfillment of other roles through the generation of resources. 


\subsection{Conceptual framework}

\subsubsection{Family Climate and Family-to-Work Enrichment}

Family climate is defined as the extent to which the family unit is sympathetic toward members need [15]. It includes family climate for sharing, family climate for sacrifice, family cohesion and family adaptability. Previous studies examined that family climate helps to reduce perceptions of family-to-work conflict through a cohesive and supportive family network. According to theory of work-family enrichment, characteristics of family should impact cross-domain enrichment [1]. Family climate provides affective ties and sense of trust among family members. Thus, cohesive family members are more likely to link their welfares with each other and conduct reciprocal and exchange behaviors. Within family, each member can get benefits and resources provided by other members. Therefore, family climate has enrichment effect on individuals' work domain. These arguments lead to the following hypothesis (Fig.1):

Proposition 1: Family climate is positively related to FWE.

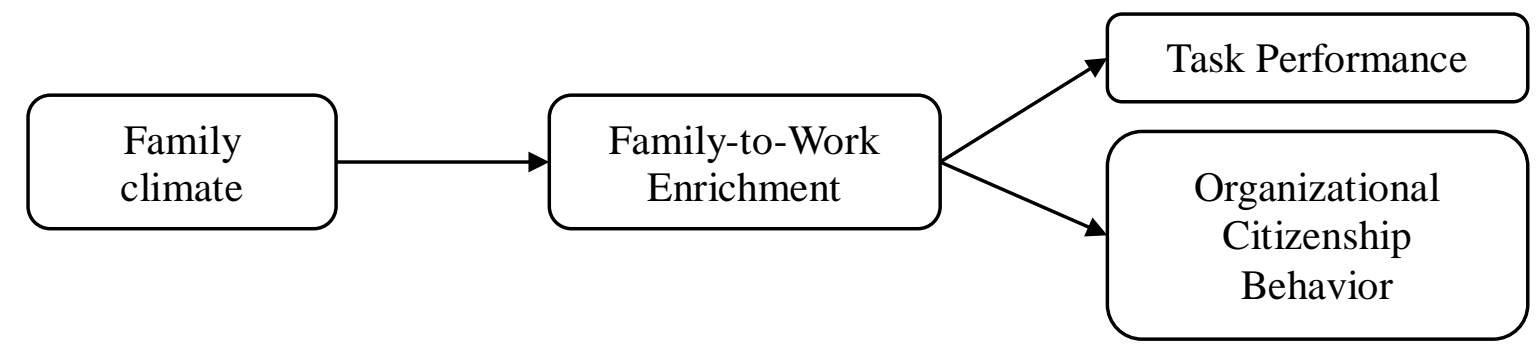

Fig.1. Conceptual framework

\subsubsection{Family-to-Work Enrichment and Job Performance}

Job performance is defined as behaviors that an employee contributes both directly and indirectly to organizational goals [16]. It consists of task performance and organizational citizenship behavior. Theory suggests that enrichment should improve performance indirectly as well as directly [13, 14]. Generally, employees enriched from family domain experience not only affective but also behavioral improvement. They should exhibit enhanced performance because they pay more efforts to the tasks and focus more on role performances. However, previous empirical results are scarce and inconclusive. For example, Witt and Carlson [17] didn't find the direct relationship between FWE and job performance. Carlson, et al. [12] examined the process by which FWE related to job performance through positive affect and job satisfaction in two distinct samples. But they found different results in two studies. In study 1, the indirect relationship was partially mediated by positive mood and fully mediated by job satisfaction. But study 2, the sample of which is supervisors didn't support the relationship. These inconsistent findings indicate that more research is needed to explore the relationship between FWE and job performance. Based on the above arguments, we propose:

Proposition 2: FWE is positively related to task performance

Proposition 3: FWE is positively related to organizational citizenship behavior

\subsubsection{Mediating Role of Family-to-Work enrichment}

To this point, we have argued that family climate promotes the enrichment from family domain to work domain, and this enrichment, in turn, translates into task performance and organizational citizenship behavior. In other words, we have implicitly described a model in which enrichment mediates the relationship between family climate and job performance. Family climate brings employees enrichment, which links to job performance. It is necessary to show how the characteristics of family system indirectly affect employees' work-related behaviors through its contribution in generating FWE. Based on these arguments, we propose:

Proposition 4a: Perceptions of FWE will mediate the positive relation between the family climate and task performance.

Proposition 4b: Perceptions of FWE will mediate the positive relation between the family 
climate and organizational citizenship behavior.

\section{Discussion}

Model proposed in this article has some contributions to the literature. First, it enriches the research of examining the positive relationship between work and family. Focusing on family's positive role, we proposed that good family climate is strongly related with employees' family-to-work enrichment, which consequently improves job performance. Second, model in this article extends the research on family-to-work enrichment by specifying the influence of family climate. Few previous studies examined the enrichment effect of family, mainly focusing on family support $[3,4]$. The proposed model focuses on the enrichment of family climate and proposed that family climate plays an important role in facilitating improvement of work domain. Third, we extend the research about family climate to individuals' interface of work and family. Past studies about family climate mainly focused on family business. We suggest that family climate can also play an important role in organization behavior and applied psychology. Finally, this article gains sight into how individuals could achieve improvement at work domain. Most previous studies suggested that individuals can be helped to achieve improvement at work by their organizations or supervisors. This article suggests that individuals can also be helped by themselves and their families to get improved.

\subsection{Implications for Future Research}

Our framework proposes that employees experiencing enrichment from family to work can improve in task performance and organizational citizenship behavior. This enrichment mainly comes from their family climates. We suggest that more research is needed to examine the validity of the proposed model. On one hand, family characteristics besides family climate can also provide individuals resources to achieve improvement in their work lives. For example, family members could provide individuals help in job search or career promotion through making good use of their social capital. Therefore, future research should explore other family characteristics that enrich employees' work domain. On the other hand, because of China's macro-environment and government policy, family climate of Chinese may be different from that of the West. For example, the open of two-child policy in China makes many couples spend more time on bringing up children. One traditional culture of Chinese is that they prefer to develop various strong ties, which play an important role in Chinese's family domain. Therefore, it is necessary to test the model in different cultural contexts.

\subsection{Limitation}

This article also has some limitations. First, we discuss only one work-related outcomes. Other work-related outcomes also exist. Second, our proposed model is limited because it cannot apply to all samples and there are always exceptions. Third, there are other family characteristics besides family climate that have enrichment effect. Finally, the proposed conceptual model needs some moderators to further understand family climate's enrichment effect on different conditions.

\section{Conclusion}

In this article, we reviewed the empirical studies on family-to-work enrichment since the year of 2006 when the theory of work-family enrichment was first proposed. We also developed a conceptual framework that identifies the relationships among family climate, family-to-work enrichment and job performance. We believe that family climate can lead to family-to-work enrichment, which leads to task performance and organizational citizenship behavior.

\section{Reference}

[1] Jeffrey H Greenhaus and Gary N Powell, When work and family are allies: A theory of 
work-family enrichment, Academy of management review, 2006 31(1) 72-92.

[2] Dawn S Carlson, K Michele Kacmar, Julie Holliday Wayne, and Joseph G Grzywacz, Measuring the positive side of the work-family interface: Development and validation of a work-family enrichment scale, Journal of Vocational Behavior, 2006 68(1) 131-164.

[3] Julie Holliday Wayne, Amy E Randel, and Jaclyn Stevens, The role of identity and workfamily support in work-family enrichment and its work-related consequences, Journal of Vocational Behavior, 2006 69(3) 445-461.

[4] Shivganesh Bhargava and Rupashree Baral, Antecedents and consequences of work-family enrichment among Indian managers, Psychological Studies, 2009 54(3) 213-225.

[5] Oi-ling Siu, Jia-fang Lu, Paula Brough, Chang-qin Lu, Arnold B Bakker, Thomas Kalliath, et al., Role resources and work-family enrichment: The role of work engagement, Journal of Vocational Behavior, 2010 77(3) 470-480.

[6] Emily M Hunter, Sara Jansen Perry, Dawn S Carlson, and Steven A Smith, Linking team resources to work-family enrichment and satisfaction, Journal of Vocational Behavior, 2010 77(2) 304-312.

[7] Laurel A McNall, Jessica M Nicklin, and Aline D Masuda, A meta-analytic review of the consequences associated with work-family enrichment, Journal of Business and Psychology, 2010 25(3) 381-396.

[8] Dawn S Carlson, Emily M Hunter, Merideth Ferguson, and Dwayne Whitten, Work-family enrichment and satisfaction: Mediating processes and relative impact of originating and receiving domains, Journal of Management, 2014 40(3) 845-865.

[9] Julie Holliday Wayne, Wendy J Casper, Russell A Matthews, and Tammy D Allen, Family-supportive organization perceptions and organizational commitment: The mediating role of work-family conflict and enrichment and partner attitudes, Journal of Applied Psychology, 2013 98(4) 606.

[10] Peter Michael Blau, Exchange and power in social life: Transaction Publishers, 1964.

[11] Stevan E Hobfoll, Social and psychological resources and adaptation, Review of general psychology, 2002 6(4) 307.

[12] Dawn Carlson, K Michele Kacmar, Suzanne Zivnuska, Merideth Ferguson, and Dwayne Whitten, Work-family enrichment and job performance: A constructive replication of affective events theory, Journal of Occupational Health Psychology, 2011 16(3) 297.

[13] Sam D Sieber, Toward a theory of role accumulation, American sociological review, 1974 567-578.

[14] Stephen R Marks, Multiple roles and role strain: Some notes on human energy, time and commitment, American sociological review, 1977 921-936.

[15] Jesse S Michel, Lindsey M Kotrba, Jacqueline K Mitchelson, Malissa A Clark, and Boris B Baltes, Antecedents of work-family conflict: A meta - analytic review, Journal of organizational behavior, 2011 32(5) 689-725.

[16] Bruce Louis Rich, Jeffrey A Lepine, and Eean R Crawford, Job engagement: Antecedents and effects on job performance, Academy of management journal, 2010 53(3) 617-635.

[17] LA Witt and Dawn S Carlson, The work-family interface and job performance: moderating effects of conscientiousness and perceived organizational support, Journal of occupational health psychology, 2006 11(4) 343. 